\title{
A comparative, randomized, controlled study on clinical efficacy and dental staining reduction of a mouthwash containing Chlorhexidine $0.20 \%$ and Anti Discoloration System (ADS)
}

Massimo Marrelli, MD',
Massimiliano Amantea, MD'1
Marco Tatullo, DDS, PhD ${ }^{1,2}$

1 Unit of Maxillofacial Surgery, Calabrodental, Crotone, Italy

2 Tecnologica Research Institute, Biomedical Section, Crotone, Italy

Corresponding author:

Marco Tatullo

Tecnologica Research Institute, Biomedical Section

Dept. of Oral Hygiene and Periodontics, Calabrodental Clinic

St. E. Fermi

88900 Crotone, Italy

E-mail: marco.tatullo@tecnologicasrl.com

\section{Summary}

Introduction. A good control of bacterial plaque is an essential factor for the success of periodontal therapy, therefore it is the main objective that the clinician together with the patient must get to have a healthy periodontium. The plaque control with mouthwashes is the most important home therapy as it helps to reduce the formation of plaque between the mechanical removal with a toothbrush.

Aim. Authors analyzed the clinical data from a trial carried out with 3 different mouthwashes containing $0.2 \%$ Chlorhexidine (CHX). In addition, the ADS (Anti Discoloration System - Curaden Healthcare) was tested in comparison with the other mouthwashes without this system.

Materials and methods. We tested antiplaque activity showed by 3 of the most commercialized mouthwashes, moreover, we tested the ability in reducing the dental staining related to the oral assumption of Chlorhexidine.

Discussion and conclusion. Our results demonstrated the clinical efficacy of the 3 mouthwashes with $\mathrm{CHX}$. Particularly performing was the anti discoloration system (Curaden Healthcare), with a clinical detection of dental stainings significantly less than the others tested. This study demonstrated the clinical efficacy of ADS system in the reduction of tooth staining, without a loss of antiplaque activity with respect to the competing mouthwashes containing $\mathrm{CHX}$.
Key words: dental plaque, oral hygiene, dental education, Chlorhexidine, dental pigmentation, dental staining.

\section{Introduction}

The plaque control is the most important aim that the clinician together with the patient must achieve to obtain an effective prevention of periodontal disease. Many studies conducted in animal models have established that the presence of pathogenic bacteria is an indispensable condition that makes possible the onset and development of gingival and periodontal diseases (1).

Additional studies have verified that the control of bacterial plaque through proper procedures of oral hygiene at home allows the complete resolution of the inflammatory process (2).

Other studies have found that a good control of bacterial plaque is an essential factor for the success of surgical and non-surgical periodontal therapy $(3,4)$.

On the contrary, Becker et al. (5) have demonstrated that without an optimal control of plaque, periodontal therapy alone is poorly effective in restoring periodontal health. This suggests that it is possible to achieve similar clinical outcomes through different surgical procedures, provided, however, that a regular and proper control of bacterial plaque is done.

The chemical control of plaque can be obtained with different pharmacological categories, including antibiotics (6) essential oils (7), and antibiotics biguanides (8).

Among the therapies commonly used to maintain control of plaque, the antiseptic Chlorhexidine ( $\mathrm{CHX})$ (9) is certainly the most studied and the most effective for the inhibition of plaque and for the prevention of gingivitis: it is recognized by more than twenty years as the gold standard for its anti-plaque and anti-bacterial activity in oral hygiene.

Some studies (10) have also verified that the constant use of $\mathrm{CHX}$ did not give rise to the development of resistant microorganisms: these results have allowed us to assert that Chlorhexidine is a safe drug, even if used for long periods.

However, even the CHX showed unfavorable characteristics, in fact, after administration of the $\mathrm{CHX}$ mouthwash, a temporary alteration of taste occurs (11); hypogeusia induced by Chlorhexidine concerns specifically salty and bitter taste.

Among the well known side effects occurring with a frequent use of $\mathrm{CHX}$, the most unaesthetic one is undoubtedly the brownish pigments that accumulate on the surfaces of teeth, as well on prosthetic crowns 
and tongue: these pigmentations restrict the use of $\mathrm{CHX}$ and patient compliance (12).

The biochemical pathways underlying the formation of pigmentations due to Chlorhexidine are the Maillard reaction with a reaction of protein denaturation: the triggering factor is represented by the interaction between food or drinks rich in chromogens after administration of Chlorhexidine.

\section{Aim of the work}

Authors analyzed data carried out from a randomized controlled clinical trial, using three products for oral hygiene, commonly sold in the dental market, with the same concentrations of $\mathrm{CHX}$ :

- Curasept mouthwash (Curaden Healthcare) $0,20 \% \mathrm{CHX}$

- Control 1 mouthwash $0,20 \% \mathrm{CHX}$

- Control 2 mouthwash 0,20\% CHX.

In addition, we evaluated whether the ADS influences the antiplaque activity of $\mathrm{CHX}$ in the formulation $0,20 \%$ compared to other formulations without ADS; finally, we evaluated the effectiveness of the ADS on the reduction of the Lobene Staining Index compared to products without ADS.

\section{Materials and methods}

We compared 3 commercially available mouthwashes: Control 1 mouthwash 0,20\% CHX, Curasept mouthwash (Curaden Healthcare, Saronno, Italy) $0,20 \% \mathrm{CHX}$, and Control 2 mouthwash $0,20 \% \mathrm{CHX}$. Our study was aimed to assess the effectiveness of $\mathrm{CHX}$ anti-plaque action by means of the clinical evaluation of periodontal indices and clinical outcomes; moreover we evaluated the presence/absence of the unaesthetic pigmentation of the tooth surfaces, usually following the administration of oral $\mathrm{CHX}$, by means of the recording of Staining-Index values.

200 subjects were recruited at the Department of "Oral Hygiene and Periodontics" of Calabrodental dental clinic (Crotone, Italy): this study was conducted among September 2011 and March 2012 and was commissioned by Curaden Healthcare.
The patients selected and involved in this study gave their signed informed consent, according with the guidelines approved by Calabrodental dental clinic (Crotone, Italy). The study and the related procedures were conducted in compliance with guidelines approved by the Calabrodental Ethics Committee (Prot. n³ July-2011/HIAP). Calabrodental ethics committee specifically approved this study. The study was conducted in compliance with the "Ethical principles for medical research involving human subjects" of Helsinki Declaration. The study was conducted in accordance with Italian laws and regulations.

\section{Study Design}

The 3 mouthwashes, Control 1 mouthwash 0,20\% $\mathrm{CHX}$, Curasept mouthwash (Curaden Healthcare, Saronno, Italy) $0,20 \% \mathrm{CHX}$, and Control 2 mouthwash $0,20 \% \mathrm{CHX}$, were marked respectively with the letters $\mathrm{C}, \mathrm{D}$ and $\mathrm{E}$ : operators and patients were not informed about the correspondence between the name of the mouthwash and the corresponding letter. In addition, the blinded mouthwashes were included in a similar packaging, with no signs or references that could indicate the name of the mouthwash: on the bottle was shown only the letter C, D or E, corresponding to the group of the same name.

The patients were mainly female (Male/Female $=$ $36 / 64 \%)$ and under 50 years old $(<50 y r s=84 \%)$ : they were randomly allocated in 3 groups named "Group C", "Group D" and "Group E"; each group was treated with the same protocol of oral hygiene but with a different mouthwash. The mean age of the patients in the 3 groups was homogeneous; moreover, it was assessed the percent of smokers in the three groups and the group $\mathrm{E}$ was the one with the higher percentage of smokers, while the group $\mathrm{C}$ showed the lowest percentage (Tab. 1).

Each patient has been identified with a different progressive code, represented by the name of the dental hygienist who has carried out the entire treatment of the patient, together with the indication of the group where the patient has been allocated after the randomization (for example: Hygienist Name-number / C-1). The recruitment was conducted according to the protocol. Exclusion criteria in this study were: absence of sys-

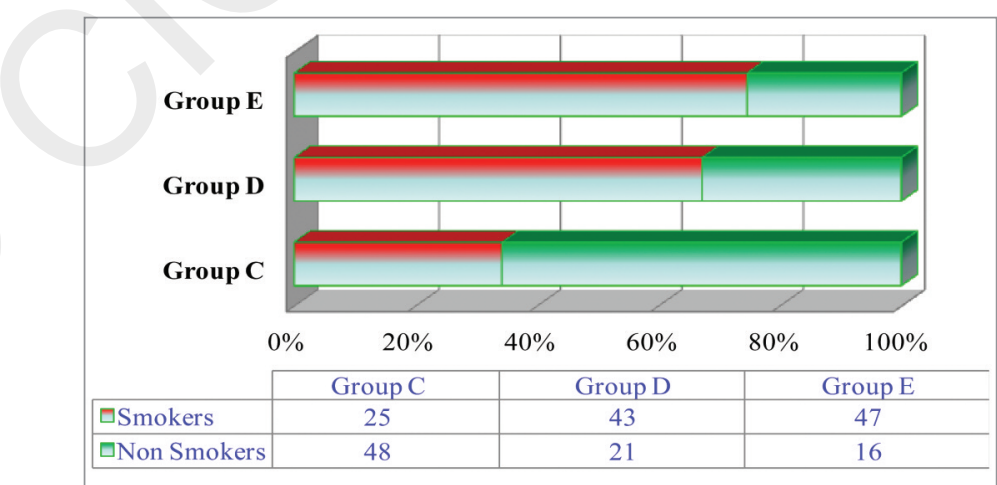


A comparative, randomized, controlled study on clinical efficacy and dental staining reduction of a mouthwash containing Chlorhexidine 0.20\% and Anti Discoloration System (ADS)

temic diseases, such as diabetes, coagulopathies, uncompensated heart diseases, vascular diseases, metabolic bone diseases, gastro-esophageal diseases allergy, etc. Patients with erosions, abrasions and abfractions of the enamel, patients affected by the bruxism and patients with mouth breathing were also excluded.

Inclusion criteria were:

1. Age \pm 18 years old

2. Non-smokers or slight smokers ( $<10$ cigarettes/day)

3. Non-drinkers or slight drinkers ( $<3$ glasses of wine/day)

4. Good compliance with the dental hygienist

The following indices were analyzed and stored:

a. Plaque Index (PI, according to Silness and Löe, 1963) (13);

b. Bleeding Index (mBI, modified Bleeding Index so to assess the presence/absence of bleeding after probing of the gingiva) (14);

c. Staining Index (SI, according to Lobene, 1968) (15);

Plaque Index was assessed according to the following clinical criteria (13):

- Value 0: Absence of bacterial plaque

- Value 1: Evident plaque by sliding the probe on the surface of the teeth

- Value 2: Visible plaque

- Value 3: Abundant plaque.

Modified Bleeding Index was assessed according to the following clinical criteria (14):

- No: Visible gingival inflammation with bleeding caused only by the passage of the probe on the gingiva

- Yes: Evident gingival inflammation with spontaneous bleeding.

The tooth is examined with a calibrate probe (diameter $0.63 \mathrm{~mm}$ ) on the lingual, mesial, distal, and buccal surfaces, and probed to test the degree of firmness. Bleeding can be more prevalent if a site is continually probed, thus it's important to insert the probe in four sites, removing the probe after the insertion. Moderate force $(<25 \mathrm{~g})$ should be used during the probing.

Staining (only area) Index was assessed according to the following clinical criteria (15):

- Value 0: Absence of pigmentations

- Value 1: Pigmentation covering up to $1 / 3$ of the region

- Value 2: Pigmentation covering $1 / 3$ to $2 / 3$ of the region

- Value 3: Pigmentation covering 2/3 of the region.

Staining (only intensity) Index was assessed according to the following clinical criteria (15):

- Value 0: No stain

- Value 1: Light pigmentations

- Value 2: Moderate pigmentation

- Value 3: Marked pigmentation.

Patients were properly visited and they were subjected to supragingival calculus removal employing ultrasonic scaler tips; polishing and tooth stains removal was also performed in the first visit $\left(T_{0}\right)$. Baseline parameters of $\mathrm{PI}, \mathrm{mBI}$ and $\mathrm{SI}$ index were properly
Table 2. Baseline data of the 3 values investigated in each of the 3 groups.

\begin{tabular}{llll}
\hline & Group C & Group D & Group E \\
\hline P. Index & m.v. 1,88 & m.v. 1,82 & m.v. 2,0 \\
B. Index & Y & Y & Y \\
S. Index & m.v. 0,68 & m.v. 0,56 & m.v. 0,59
\end{tabular}

Legend:

m.v. : mean value

Y: yes (according to $\mathrm{mB}$.Index described in the Methods section)

recorded before the previously described first oral hygiene treatment $\left(T_{0}\right)$ (Tab. 2). The analyzed data showed a correct randomization. Follow-up evaluations were performed after 7 days $\left(T_{1}\right)$ and after 14 days $\left(T_{2}\right)$ from the first visit $\left(T_{0}\right)$. Photographic documentation was archived at baseline, $T_{0}, T_{1}$ and $T_{2}$.

In order not to influence the formation of the pigmentations, the patients were advised to limit the chewing and drinking of chromogenic foods such as tea, coffee, red wine, spinach, during the 15 days of duration of the study: every assumption of these substances was to be reported in the appropriate schedule provided them in the $T_{0}$ visit.

\section{"Reverse" motivation}

During the study, patients were required not to use the daily mechanical oral hygiene, while they were required only to rinse with $10 \mathrm{ml}$ of solution of the assigned mouthwash, twice a day, not before 30 minutes from their meals, for a duration of about 60 seconds.

The reverse motivation finds its rational application in our study, and was reported in literature in a study by Ross et al. (16), in which subjects who had heard messages that dissuaded to perform the oral hygiene, showed a significantly more negative attitude toward this practice and remembered fewer episodes in which they had washed their teeth.

\section{Statistical analysis}

The collected data were analyzed by means of the statistical software SPSS/PC version 10.1 for Windows. The statistical evaluations were carried out by means of Student's t test for paired samples 2-tailed. The results were obtained by comparing, for each group of patients analyzed, the values detected at time $T_{1}$ and the values measured at time $\mathrm{T}_{2}\left[\mathrm{~T}_{1} v s \mathrm{~T}_{2}\right]$. The test was considered significant with $p$-value $<0.05$.

\section{Results}

At the end of the study, data were analyzed from many points of interest, and results were investigated per groups and per single value. 


\section{P. Index}

The P. Index distribution in the 3 groups appears substantially homogeneous with the exception of Group $\mathrm{E}$, in which we detected few patients with $\mathrm{P}$. Index values between 2 and 3 .

P. Index values show that the Group $C$ has an increase of 0.03 points/media from $T_{1}$ to $T_{2}$; Group $D$ shows a clear reduction of $P$. Index values, a decrease of 0.26 points/media. Group $E$ is the only one with very high values if compared with the other two groups, with a decreasing of points/media of just 0.06; however, the Group E is also the one who started with mean values of P.Index equal to 2 before the initial hygiene performed in $T_{0}$; therefore, it is supposed that the patients allocated in Group D are less prone to plaque control. It should be noted that the Group D starts with a P. Index at baseline equal to 1.82 and after 7 days it has a $P$. Index equal to 1.7 , which constantly tends to stabilize itself at lower values (1.44) in the following days (Tab. 3).

After the data evaluation, we can assert that $P$. Index variations detected in the 3 Groups $C, D$ and $E$ are not statistically significant, with the exception of Group D which shows a high significance. This data allows us to observe a better clinical outcome showed by mouthwash with ADS, in relation to the control of dental plaque.

\section{mB. Index}

The modified B. Index values of the Group C show that the bleeding persists even after the first week of treatment with the mouthwash $\mathrm{C}$, however, starting from the second week the leakage of blood stops. The Group D shows an absence of bleeding at the first follow-up and this finding remains unaltered even during the final visit at $T_{2}$. The Group $E$ is the only one that does not seem to benefit from the bacterio-

Table 3. Comparison between $T_{1}$ and $T_{2}$ data of the 3 values investigated in each of the 3 groups.

\begin{tabular}{lll}
\hline & $\mathbf{T}_{\mathbf{1}}$ & $\mathbf{T}_{\mathbf{2}}$ \\
\hline Group C & & \\
\hline P.Index & 1,82 & 1,85 \\
P.Index ( $\geq 2)$ & 2,09 & 2,07 \\
B.Index & $\mathrm{Y}$ & $\mathrm{N}$ \\
S.Index & 0,52 & 0,81 \\
\hline Group D & & \\
\hline P.Index & 1,7 & 1,44 \\
P.Index ( $\geq 2)$ & 2,1 & 2 \\
B.Index & $\mathrm{N}$ & $\mathrm{N}$ \\
S.Index & 0,21 & 0,49 \\
\hline Group E & & \\
\hline P.Index & 2,1 & 2,04 \\
P.Index ( $\geq 2)$ & 2,04 & 2,04 \\
B.Index & $\mathrm{Y}$ & $\mathrm{Y}$ \\
S.Index & 0,3 & 0,64 \\
\hline
\end{tabular}

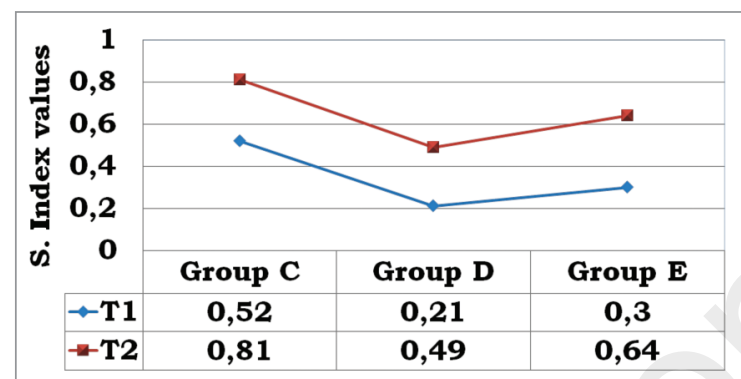

Table 4. Comparison of S.Index mean values between $T_{1}$ and $T_{2}$ in the 3 groups.

static and anti-plaque action of $\mathrm{CHX} 0.20 \%$; however, this specific result may also depend in substantial part by the clinical condition at baseline of the Group E, as previously stated (Tab. 3).

\section{S. Index}

S. Index data in the 3 groups show that the Group C presents an increase in the average value of the staining index computed in 0.29 points/media, between the control at 7 days and the control at 15 days. Group E, instead, shows an increasing in the average value of S.Index of 0.34 points/media, when you compare the control values at 7 days and the control values at 15 days; however, the detected average value of S.Index at 14 days is lower than the one observed in the Group C. Finally, the Group D shows a variation of the average value of $\mathrm{S}$. Index assessed in 0.28 points $/ \mathrm{me}$ dia between the control at 7 days and the control at 15 days, however, it's important to emphasize that the absolute values at $\mathrm{T}_{2}$ control reveal a pigmenting ability of Curasept mouthwash significantly lower than the other tested mouthwashes: this mouthwash has a formulation containing the anti discoloration system (ADS) that has just the function of reducing the dental pigmentations (Tabs. 3, 4).

\section{Discussion}

Oral care is an essential part of the general health of the population. Dental bacterial plaque has been related to several systemic diseases such as pneumonia and endocarditis (17). The development of oral diseases is often associated with the composition and the quantity of dental plaque, with the diet, and with the typology of the salivary flow (18).

$\mathrm{CHX}$ has been investigated for over 30 years as an antimicrobial agent for the chemical control of plaque formation. Different concentrations are available on the market, mainly ranging from 0.02 to $0.3 \%$; however, recently, the Food and Drugs Administration (FDA) introduced high concentration solution (3.5\%) to be diluted with water before use. As shown by in vivo studies (19), the mechanism of action of the Chlorhexidine seems to be dose-dependent: bacterio- 
static at very low concentrations $(0.02-0.06 \%)$, and bactericidal at higher concentrations $(0.12-0.20 \%)$.

FDA suggested a use of $\mathrm{CHX}$ (at 0.12 and $0.2 \%$ concentrations) administered as oral rinses of $10-15 \mathrm{~mL}$, for about 30 seconds, for a limited period of time (respectively, 1 month or 2 weeks) $(20,21)$.

$\mathrm{CHX}$ would appear to have dose-dependent effects also in terms of its most common local adverse effects: dysgeusia and tooth pigmentation (22). Because of this, different concentrations have been suggested over time, trying to balance beneficial and adverse effects of $\mathrm{CHX}$, in order to improve the patient compliance.

As recently reported in the literature $(23,24)$, the use of mouthwashes containing $0.2 \%$ of $\mathrm{CHX}$ showed a substantial reduction of gingival inflammation and plaque indexes $(23,24)$. Several studies demonstrated that subjects that performed their oral care with mouthwashes containing $0.2 \%$ of $\mathrm{CHX}$ improved significantly their P. Index (25), the B. Index $(26,27)$, and the S. Index (28).

Recently, it has been investigated the possibility of reducing brown pigmentations associated with the use of $\mathrm{CHX}$-based products, particularly the mouthwashes (29), by interrupting the Maillard reaction. To date, the efficacy of antiseptic solutions containing $0.20 \% \mathrm{CHX}$ has been consistently carried out by some peer-reviewed publications (30-32), and according to these studies, it was highlighted that $\mathrm{CHX}$ products with effective anti-staining additives, led to a reduction of $\mathrm{CHX}$ antimicrobial activity (33).

In our work we have analyzed the antiplaque activity of 3 of the most frequently used mouthwashes on the market, moreover, we have assessed their ability to avoid the typical pigmentations occurring after a lasting of oral $\mathrm{CHX}$ treatment with a duration of several days.

We compared the three mouthwashes. Patients treated with Curasept mouthwash, based on a formulation with ADS, showed a fairly constant antiplaque activity, compared to baseline values stored in this research. This finding is fully confirmed by the subanalysis performed in patients with full-mouth $P$. Index $\geq 2$ : in fact, even into this sub-group, we can observe a noticeable reduction of the plaque index in Curasept group; although the observed plaque control in this group is not clinically satisfactory, we must consider the concomitant relative absence of mechanical oral hygiene required by the "reverse motivation" described in the methods section, as required by the protocol.

We evaluated the effectiveness of the ADS on the reduction of the $S$. Index. In the Group C (Control 1) the average value of $S$. Index resulted increased between the control at 7 days and at 15 days. In the group E (Control 2) we observed a similar trend, with average $S$. Index values increased between the $\mathrm{T}_{1}$ and the $\mathrm{T}_{2}$. Finally, the Group $\mathrm{D}$ (treated with Curasept) showed some variations of the average $S$. Index values, revealing that Curasept mouthwash has a lower pigmenting ability, with respect to the other tested mouthwashes.
Although $\mathrm{CHX}$ is unanimously recognized as one of the most effective cationic antiseptic compound currently used in medical practice (34), different research groups have investigated the antiplaque action of other mouthwash products containing different principles rather than $\mathrm{CHX}$ (36). All these works failed to find an alternative antiplaque solution as effective as $\mathrm{CHX}$, such as Listerine (without $\mathrm{CHX}$ ) which has been demonstrated to have a significantly lower antimicrobial activity than $\mathrm{CHX}$-based products (35).

Notwithstanding, some studies demonstrated that $\mathrm{CHX}$ elicits a low efficacy in the treatment of severe periodontal diseases (36), or in the presence of coronal caries (37), if it is not associated to a mechanical removal of bacterial plaque. It is likewise important to emphasize that in the presence of advanced periodontal disease the use of $\mathrm{CHX}$ could result not sufficient to improve the periodontal condition, if not associated with subgingival treatment (38).

On the other hand, it has been demonstrated the possibility of interaction and mutual inactivation of chlorhexidine and toothpaste that contains a common detergent compound, sodium lauryl-sulfate (38). Therefore, we recommend the use of $\mathrm{CHX}$ after an interval of 30 minutes by the use of other products for oral hygiene.

Previous works evidenced also an antagonist action of $\mathrm{CHX}$ with monofluorophosphate content in some mouthwashes (39).

Gusolley (40) provided in the 2010 a ranking of the antiplaques and antigingivitis effects of the three mouthwashes containing $\mathrm{CHX}$, essential oils (EOs) and cetypyridinium chloride (CPC). Among these three, EOs was reported as the second best antiplaque and anti-inflammatory mouthwash right after $\mathrm{CHX}$ (41).

Phenols and essential oils have been used for a long time to rinse the oral cavity. One of the main products is Listerine, made of essential oils, thymol and eucalyptol combined with alcohol-based menthol. Although this product is not as effective as chlorhexidine (34) it obtained significant reductions in the level of plaque and gingivitis in various studies. EOs were also demonstrated to represent a reliable alternative to chlorhexidine mouthwash in long-term use (41).

Antiplaque and antimicrobial mouth rinses used thus far in periodontal practice also contain ingredients such as alcohol or sugar (42). These ingredients enhance the cariogenic potential of the substrate and promote halitosis. A recent study evaluated the effects of the use of an herbal mouth rinse on oral hygiene improvement (43). In this study emerged that the Indian herb Triphala (44-46) exhibits similar antiplaque efficacy to that of $\mathrm{CHX}$ and was more effective in inhibiting plaque formation with lesser or no side effects (47).

Together with EOs, CHX is still considered the gold standard against plaque formation (48). $\mathrm{CHX}$ is also available in gel at different concentrations ranging from 1 to 0.2 and $0.12 \%$ (49); studies proved the antibacterial activity of $\mathrm{CHX}$ gels at the high concentrations available $(2 \%, 1 \%)$ while at the lower concentrations $(0.2 \%) \mathrm{CHX}$ gel action resulted inhibited by dentin matrix and type I collagen (50). Furthermore, 
some studies reveal that the gel cannot reach all surfaces of the tooth.

Alternatively, instead of rinse, there is a spray (18) that allows for convey the compound directly on the surfaces of the teeth making a dosage, normally lower than that used in mouthwashes, effective. Spray formulations would appear to be particularly indicated in patients with mental and physical impairments, leading to a significant reduction in plaque and gingival bleeding indexes (51-53).

\section{Conclusions}

Our trial has clinically showed, once again, the efficacy of chlorhexidine mouthwashes. All mouthwashes analyzed showed an action not only in reducing the ability of plaque formation in the absence of brushing, but also in the protection of the gingival tissues, which showed a less erythematous appearance, and with a lower index of spontaneous bleeding. But, the major and significant evidence we had it in assessing the optimal response of the system ADS, by Curasept, to the formation of pigmentation on tooth enamel. This response has allowed to combine aesthetics and antiplaque action, without reducing the effectiveness of each of them in oral hygiene at home.

\section{Consent Statement}

Written informed consent was obtained from the patient for publication of this case report and accompanying images. A copy of the written consent is available for review by the Editor-in-Chief of this journal.

\section{Availability of supporting data}

A copy of all the supporting data of this study is available for everyone who requests it.

\section{Competing Interests}

The Authors declare that they have no competing interests.

\section{Authors' contributions}

MM and MA: ideated, directed and conducted the study protocol.

MT: drafted the manuscript and participated to all the research activities.

\section{Acknowledgements}

The Authors would like to thank the following people: Dr. Stefano Giovannardi and Dr. Restituta Castellac- cio, Curaden Healthcare, for their precious contribution to this clinical research;

Dr. Marta Giovannardi, Curaden Healthcare, for her valuable contribute to the study design and to the critical analysis of the outcomes;

Dr. Roberto Cimmino, Curaden Healthcare, for his kind support in the organizations of the research activities;

Dr. Dora Marrelli, Dr. Sandro Sestito, Dr. Domenico Tarsitano, Department of Oral Hygiene and Periodontics of Calabrodental Clinic, for their hard and professional work in the collection of the data.

A sincere thank you to the Department of Oral Hygiene and Periodontics of Calabrodental Clinic, to all the administrative area and to all the operators of Marrelli Group that participated to the organization of the research activities.

\section{List of Abbreviations}

Anti Discoloration System (ADS)

Chlorhexidine ( $\mathrm{CHX})$

Plaque Index (PI)

modified Bleeding Index ( $\mathrm{mBI})$

Bleeding Index (BI)

Staining Index (SI)

Time 0, 1, $2\left(T_{0}, T_{1}, T_{2}\right)$

Essential oils (EOs)

Cetypyridinium chloride (CPC)

\section{References}

1. Quirynen M, Bollen CM, Vandekerckhove BN, Dekeyser C, Papaioannou W, Eyssen H. Full-vs. partial-mouth disinfection in the treatment of periodontal infections: short-term clinical and microbiological observations. J Dent Res. 1995 Aug;74(8):1459-67.

2. Pongnarisorn NJ, Gemmell E, Tan AE, Henry PJ, Marshall RI, Seymour GJ. Inflammation associated with implants with different surface types. Clin Oral Implants Res. 2007 Feb;18(1):114-25.

3. Drisko $\mathrm{CH}$. Nonsurgical periodontal therapy. Periodontol. 2000. 2001;25:77-88.

4. Heitz-Mayfield LJ, Lang NP. Surgical and nonsurgical periodontal therapy. Learned and unlearned concepts. Periodontol. 2000. 2013 Jun;62(1):218-31. Review.

5. Becker W, Becker BE, Caffesse R, Kerry G, Ochsenbein C, Morrison E, Prichard J. A longitudinal study comparing scaling, osseous surgery, and modified Widman procedures: results after 5 years. J Periodontol. 2001 Dec;72(12):167584.

6. Mohan RR, Doraswamy DC, Hussain AM, Gundannavar G, Subbaiah SK, Jayaprakash D. Evaluation of the role of antibiotics in preventing postoperative complication after routine periodontal surgery: A comparative clinical study. J Indian Soc Periodontol. 2014 Mar;18(2):205-12.

7. Marchetti E, Mummolo S, Di Mattia J, Casalena F, Di Martino S, Mattei A, Marzo G. Efficacy of essential oil mouthwash with and without alcohol: a 3-day plaque accumulation model. Trials. 2011 Dec 15;12:262.

8. Ennibi O, Lakhdar L, Bouziane A, Bensouda Y, Abouqal R. Chlorhexidine alcohol base mouthrinse versus Chlorhexidine 
A comparative, randomized, controlled study on clinical efficacy and dental staining reduction of a mouthwash containing

formaldehyde base mouthrinse efficacy on plaque control: double blind, randomized clinical trials. Med Oral Patol Oral Cir Bucal. 2013 Jan 1;18(1):e135-9.

9. Varoni E, Tarce M, Lodi G, Carrassi A. Chlorhexidine $(\mathrm{CHX})$ in dentistry: state of the art. Minerva Stomatol. 2012 Sep; 61(9):399-419.

10. Schlett CD, Millar EV, Crawford KB, Cui T, Lanier JB, Tribble DR, Ellis MW. Prevalence of chlorhexidine-resistant methicillin-resistant Staphylococcus aureus following prolonged exposure. Antimicrob Agents Chemother. 2014 Aug;58(8):4404-10. doi: 10.1128/AAC.02419-14.

11. Marinone MG, Savoldi E. Chlorhexidine and taste. Influence of mouthwashes concentrate on andof rinsing time. Minerva Stomatol. 2000 May;49(5):221-6.

12. Eriksen HM, Nordbø H, Kantanen H, Ellingsen JE. Chemical plaque control and extrinsic tooth discoloration. A review of possible mechanisms. J Clin Periodontol. 1985 May; 12(5):345-50. Review.

13. Löe H, Silness J. Periodontal disease in pregnancy. I. Prevalence and severity. Acta Odontol Scand. 1963; 21:533-551.

14. Löe H. The Gingival Index, the Plaque Index and the Retention Index System. J Periodontol. 1967;38:610- 616.

15. Lobene RR. Effect of dentifrices on tooth stains with controlled brushing. JADA. 1968;77:849-855.

16. Ross M, McFarland C, Fletcher G J. The effect of attitude on the recall of personal histories. Journal of Personality and Social Psychology. 1981;40:627-634.

17. Ravald N, Hamp SE, Birkhead D. Long-term evaluation of root surface caries in periodontally treated patients. J Clin Periodontol. 1986; 13:758-767.

18. Puig Silla M, Montiel Company JM, Almerich Silla JM. Use of chlorhexidine varnishes in preventing and treating periodontal disease. A review of the literature. Med Oral Pato Cir Bucal. 2008; 13:E257-60.

19. Jenkins S, Addy M, Wade W. The mechanism of action of chlorhexidine. A study of plaque growth on enamel inserts in vivo. J Clin Periodontol. 1988;15:415-24.

20. www.drugs.com. Chlorhexidine- FDA Official Information, side effect and Use. www.drugs.com; 2011. Available at: http://www.drugs.com/pro/chlorhexidine.html

21. Ellepola AN, Samaranayake LP. Adjunctive use of chlorhexidine in oral candidoses: a review. Oral Dis. 2001;7:11.

22. Hoffmann T, Bruhn G, Richter S, Netuschil L, Brecx M. Clinical controlled study on plaque and gingivitis reduction under long-term use of low-dose chlorhexidine solutions in a population exhibiting good oral hygiene. Clin Oral Investig. 2001;5:89-95.

23. Berchier CE, Slot DE, Van der Weijden GA. The efficacy of $0.12 \%$ chlorhexidine mouthrinse compared with $0.2 \%$ on plaque accumulation and periodontal parameters: a systemic review. J Clin Periodontol. 2010;37:829-39.

24. Matthews D. No difference between $0.12 \%$ and $0.2 \%$ chlorhexidine mouthrinse on reduction of gingivitis. Evid Based Dent. 2011;12:8-9.

25. Grossman E, Reiter G, Sturzenberg OP, Rosa M, Dickinson TD, Flrretti GA, et al. Six-month study of the effects of a chlorhexidine mouthrinse on gingivitis in adults. J Periodontal Res. 1986;21(s16):33-43.

26. Gunsolley JC. A meta-analysis of six-month studies of antiplaque and antigingivitis agents. J Am Dent Assoc. 2006; 137:1649-57.

27. Chaves ES, Kornman KS, Manwell MA, Jones AA, Newbold DA, Wood RC. Mechanism of irrigation effects on gingivitis. J Periodontol. 1994;65:1016-21.

28. Russell AD. Chlorhexidine: antibacterial action and bacterial resistance. Infection. 1986;14:212-5.

29. Stookey GK, Beiswanger B, Mau M, Isaacs RL, Witt JJ, Gibb R. A 6-months clinical study assessing the safety and effi- cacy of two cetylpyridinium chloride mouthrinses. Am J Dent. 2005;18 spec No:24A-8A.

30. Addy M, Sharif N, Moran J. A non-staining chlorhexidine mouthwash? Probably not: a study in vitro. Int J Dent Hyg. 2005;3:59-63.

31. Cortellini P, Labriola A, Zambelli R, Prato GP, Nieri M, Tonetti MS. Chlorhexidine with an anti discoloration system after periodontal flap surgery: a cross-over, randomized, triple-blind clinical trial. J Clin Periodontol. 2008;35:614-20.

32. Bernardi F, Pincelli MR, Carloni S, Gatto MR, Montebugnol L. Chlorhexidine with an anti discoloration system. A comparative study. Int J Dent Hyg. 2004;2:122-6.

33. Solis C, Santos A, Nart J, Violant D. $0.2 \%$ chlorhexidine mouthwash with an antidiscoloration system versus $0.2 \%$ chlorhexidine mouthwash: a prospective clinical comparative study. J Periodontol. 2011;82:80-5.

34. Guggenheim B, Meier A. In vitro effect of chlorhexidine mouth rinses on polyspecies biofilms. Schweiz Monatsschr Zahnmed. 2011;121(5):432-41.

35. Mangram AJ, Horan TC, Pearson ML, Silver LC, Jarvis WR Guideline for prevention of surgical site infection, 1999. Hospital Infection Control Practices Advisory Committee. Infect Control Hosp Epidemiol. 1999;20:250-78.

36. Goutham BS, Manchanda K, Sarkar AD, Prakash R, Jha K, Mohammed S. Efficacy of two commercially available Oral Rinses - Chlorohexidine and Listrine on Plaque and Gingivitis - A Comparative Study. J Int Oral Health. 2013 Aug;5(4):5661. Epub 2013 Aug 28.

37. Medaiah S, Srinivas M, Melath A, Girish S, Polepalle T, Dasari AB. Chlorhexidine chip in the treatment of chronic periodontitis: a clinical study. J Clin Diagn Res. 2014; Jun;8(6):ZC22-5.

38. Papas AS, Vollmer WM, Gullion CM, Baber J, Laws R, Fellows J, Hollis JF, Maupomé G, Singh ML, Synder J, Blanchard $\mathrm{P}$ and the PACS Collaborative Group. Efficacy of chlorhexidine varnish for the prevention of adult caries: a randomized trial. J Dent Res. 2012; 91(2):150-155.

39. Barkvoll P, Rolla G, Svendsen K. Interaction between chlorhexadine digluconate and sodium lauryl sulfate in vivo. J Clin Periodontol. 1989 Oct 16(9):593-5.

40. Barkvoll P, Rolla G, Bellagamba S. Interaction between chlorhexidine digluconate and sodium monofluorophosphate in vitro. Scand J Dent Res. 1988 Feb;96(1):30-3.

41. Gunsolley JC. Clinical efficacy of antimicrobial mouth-rinses. J Dent. 2010 Jun;38 suppl 1:S6-10.

42. Van Leeuwen MP, Slot DE, Van der Weidjen GA. Essential oils compared to chlorhexidine with respect to plaque and parameters of gingival inflammation: a systemic review. J Periodontol. 2011;82(2):174-94.

43. Leyes Borrajo JL, Garcia VL, Lopez CG, Rodriguez-Nunez I, Garcia FM, Gallas TM. Efficacy of chorhexidine mouthrines with and without alcohol: a clinical study. J Periodontol. 2002;73:317-321.

44. Nagappan N, John J. Antimicrobial efficacy of herbal and clorhexidine mouth rinse: a systematic review. J Dent Med Sci. 2012;2:5-10.

45. Desai A, Anil M, Debnath A. A clinical trial to evaluate the effects of triphala as a mouthwash in comparison with clorhexidine in chronic generalized periodontitis patient. Indian J Dent Adv. 2010;2:243-7.

46. Maurya DK, Mittal N, Sharma KR, Nath G. Role of triphala in the management of periodontal disease. Anc Sci Life. 1997;17:120-7.

47. Bajaj N, Tandon S. The effect of triphala and clorhexidine mouthwash on dental plaque, gingival inflammation, and microbial growth. Int J Ayurveda Res. 2011;2:29-36.

48. Narayan A, Mendon C. Comparing the effect of different mouth-rinses on the novo plaque formation. J Contemp Dent Res Pract. 2012;13:460-3. 
49. Jones CG. Chlorhexidine: is it still the gold standard? Periodontol. 2000. 1997; 15:55-62.

50. Prabhakar AR, Taur S, Hadakar S, Sugandhan S. Comparison of antibacterial efficacy of calcium hydroxide paste $2 \%$ chlorhexidine gel and turmeric extract as an intracanal medicament and their effect on microhardness of root dentin: an in vitro study. Int J Clin Pediatr Dent. 2013 Sep;6(3):171-7.

51. Porteneir J, Haapsolon, Ostrovik D, Yamauchi M, Haapasalo $M$. Inactivation of the antibacterial activity of iodine potassium iodide and chlorhexidine digluconate against Enterococcus faecalis by dentin, dentin matrix, type-I collagen and heat killed microbial whole cell. J Endod. 2002;28(9):634-637.

52. Montiel-Company JM, Almerich-Silla JM. Efficacy of two antiplaque and antigingivitis treatments in a group of mentally retarded patients. Med Oral. 2002;7:136-43.

53. Chibinski ACR, Pochapski MT, Farago PV, Santos FA, Czlusniak GD. Clinical evaluation of chlorhexidine for the contro of dental biofilm in children with special needs. Community Dent Health. 2011;28:222-6. 